\title{
Cárie dentária e condições sócio-econômicas: um estudo transversal com jovens de 18 anos de Florianópolis, Santa Catarina, Brasil
}

\author{
Dental caries and socioeconomic conditions: \\ a cross-sectional study among 18 years-old male \\ in Florianópolis, Santa Catarina State, Brazil
}

Evel ise Ribeiro Gonçalves 1

Marco Aurélio Peres 2

Wagner Marcenes 3

\footnotetext{
15357 Fjell, Bergen, Norway.

2 Departamento de Saúde

Pública, Universidade Federal de Santa Catarina. Campus Universitário Trindade, Florianópolis, SC 88010-970, Brasil. peresp@repensul.ufsc.br

3 Royal Free and University College, London Medical School, Department of Epidemiology and Public Health. 1-19 Torrington Place, London, WC1E 6BT, UK.
}

\begin{abstract}
The aim of this study was to assess the prevalence and severity of dental caries and need for treatment among 18 years-old males in Florianópolis, Southern Brazil. In addition, the associations between dental caries and socioeconomic conditions were tested. A cross sectional study was carried out. A random sample of 300, was sel ected from a list of Brazilian Army conscripts. Clinical data were collected according to World Health Organization criteria. Soci oeconomic data (years of education of the subjects, their fathers and mothers and family income) were collected through interviews. The statistical significance of associations between soci oeconomic indicators and dental caries prevalence were tested using the chi-square test whilst for severity of dental caries Mann-Whitney test was used. The prevalence of dental caries was $81 \%$ and the mean DMF-T was 4.5. The mean number of teeth that needed treatment was 1.2. Both dental status and treatment need were statistically significantly associated with soci oeconomic indicators. Those with low levels of education and income experienced more disease and needed more treatment than those from high levels of education and income.
\end{abstract}

Key words Dental Caries; Social Conditions; Adolescence; Oral Health; Epidemiology

Resumo Os objetivos deste estudo foram conhecer a prevalência e severidade da cárie dentária, bem como necessi dades de tratamento odontológi co, al ém de testar sua associação com variáveis sóci o-econômicas em jovens de 18 anos de idade do sexo masculino em Florianópolis, Santa Catarina, Brasil. Foi realizado um estudo transversal em uma amostra aleatória de alistandos ( $n=300$ ) do Exército Brasilei ro. Foram utilizados os critérios de diagnóstico da Organi zação Mundial da Saúde. Utilizou-se um questionário sócio-econômi co para se aferir o grau de escolaridade do alistando, de seus pais e a renda familiar. Associações entre a prevalência de cárie, o índi ce CPO-D e seus componentes e as variáveis sócio-econômi cas foram avaliadas por meio dos testes qui-quadrado e Mann-Whitney, respecti vamente. A prevalência de cárie foi de $81 \%$ eo índice CPO-D médi o foi igual a 4,6. Apenas 1,2 dente por pessoa, em média, apresentou-se com necessidade de tratamento. Diferenças estatisticamente significati vas foram encontradas na prevalência e severi dade de cárie (CPO-D), sendo os pi ores indi cadores verificados nos grupos de menor escolaridade e renda, indi cando serem estes grupos prioritári os para medidas preventivas e assistenciais.

Palavras-chave Cárie Dentária; Condições Sociais; Adolescência; Saúde Bucal; Epi demiologia 


\section{Introdução}

Apesar da cárie dentária ser a doença bucal mais estudada em todo o mundo, a maioria dos estudos concentra-se em crianças em idade escolar, não havendo pesquisas suficientes sobre a situação da doença em adultos jovens (Truin et al., 1993). Os poucos estudos epidemiológicos de cárie em adultos jovens foram realizados quase que exclusivamente nos países desenvolvidos (Tabela 1 ).

A falta de informações epidemiológicas básicas em adultos jovens é uma limitação séria por dois motivos principais. Primeiro, é importante conhecer o comportamento da doença em todas as idades, permitindo assim seu monitoramento epidemiológico. Segundo, com a redução da prevalência e severidade da cárie em crianças observadas no país, aumentaram as possibilidades de expansão da cobertura de serviços para outros grupos populacionais, incluindo-se os adultos jovens. A epidemiologia é um instrumento útil para o planejamento de serviços.

Os objetivos do presente estudo foram conhecer a prevalência, severidade da cárie e necessidades de tratamento odontológico na população de 18 anos de Florianópolis, Santa Catarina, além de testar as associações destas com as condições sócio-econômicas da população estudada. Somado a outro estudo sobre as doenças periodontais (Gesser et al., 2001) realizado na mesma população tem-se um amplo quadro epidemiológico das condições de saúde bucal aos 18 anos de idade no município.

\section{Métodos}

Foi realizado um estudo transversal para cárie e doença periodontal cuja população de referência correspondeu aos 3.452 alistandos do Exército Brasileiro em Florianópolis no ano de 1999. Para a obtenção do tamanho mínimo da amostra de 267 pessoas, considerou-se a prevalência de $86 \%$, um erro amostral de $2 \%$ e um nível de confiança de $95 \%$. O valor de prevalência utilizado para o cálculo foi o relativo à doença periodontal na Região Sul do Brasil, para a faixa etária entre 15 e 19 anos (MS, 1988).

Como foram estudadas cárie e doença periodontal na mesma população, adotou-se o maior tamanho da amostra, o obtido para estimar a prevalência de doença periodontal. Foram acrescidos mais 33 indivíduos considerando-se as possíveis perdas, totalizando, portanto, trezentos indivíduos.
Sortearam-se aleatoriamente dez dias para a realização da pesquisa, dentre os trinta dias destinados aos exames médicos e odontológicos de rotina do Exército. Em cada um dos dias da pesquisa, sortearam-se trinta jovens, utilizando-se o processo de amostragem casual simples.

Os dados sócio-econômicos foram coletados por meio de um questionário que incluiu os anos de estudo do alistando, do seu pai e da sua mãe, além da renda familiar, em reais, obtida no mês anterior à entrevista. A renda familiar foi convertida posteriormente em salários mínimos - SM ( 1 SM =R\$136,00 ou US\$ 73.50 - valor em dólares, segundo câmbio da época da pesquisa).

Os dados clínicos foram coletados utilizando-se os critérios preconizados pela Organização Mundial da Saúde (OMS) em sua quarta versão do Oral Health Surveys - Basic Methods (WHO, 1997).

Uma única dentista (E. R. G.) realizou todos os exames referentes às condições dentárias e necessidades de tratamento, auxiliada por um anotador, também cirurgião-dentista. Este último realizou os exames acerca das condições periodontais, relatados em outra publicação (Gesser et al., 2001). Utilizaram-se espelhos bucais planos e sondas periodontais (CPI probe) esterilizados, e foram seguidas as normas de biossegurança.

Exercícios de calibração, pré-teste do questionário e estudo-piloto foram realizados previamente ao estudo com trinta jovens da mesma idade não incluídos na amostra.

Realizaram-se exames em duplicata em dez por cento da amostra utilizando-se a estatística kappa para o cál culo da reprodutibilidade, tomando-se o dente como unidade de análise para a aferição da confiabilidade de diagnósti$\mathrm{co}$, seguindo metodologia anteriormente descrita (Peres et al., 2001). Foi obtido o consentimento de cada um dos alistandos para a realização da pesquisa.

A entrada dos dados e análises estatísticas foram realizadas com o programa SPSS 10.0 (Nie et al., 1975). Para testar as diferenças entre proporções e diferenças no índice CPO-D em relação às variáveis sócio-econômicas, foram empregados o teste do qui-quadrado e o MannWhitney test respectivamente. A adoção de testes não paramétricos justifica-se, pois o índice de cárie utilizado (CPO-D) não apresenta distribuição normal. Foi adotado o nível de significância estatística de 5\%. 
Estudos epidemiológicos de cárie dentária em adultos jovens. Autores e ano da publicação, ano do estudo, idade ou faixa etária estudada, tamanho da amostra, CPO-D médio e país.

\begin{tabular}{|c|c|c|c|c|c|}
\hline Autores & Ano do estudo & $\begin{array}{c}\text { Idade/ } \\
\text { Faixa etária }\end{array}$ & $\begin{array}{c}\text { Tamanho } \\
\text { da amostra }\end{array}$ & CPO-D médio & País \\
\hline Dale, 1969 & 1969 & 18 & 106 & 18,4 & Austrália \\
\hline DePaola, 1983 & 1982 & 18 & 32 & 9,0 & Estados Unidos \\
\hline \multirow[t]{2}{*}{ Von der Fehr, 1982} & 1968 & $17-20$ & ND & 20,0 & Noruega \\
\hline & 1978 & & & 17,0 & \\
\hline Truin et al., 1993 & 1986 & $15-19$ & 530 & 6,6 & Holanda \\
\hline MS, 1988 & 1986 & $15-19$ & 4.798 & 12,7 & Brasil \\
\hline Richardson \& Mclntyre, 1996 & 1988 & 18,9 & 416 & 7,3 & Inglaterra \\
\hline Morgan et al., 1992 & 1988 & $15-19$ & 1.100 & 4,33 & Austrália \\
\hline Gaare et al., 1989 & 1989 & $22-25$ & 201 & 3,0 & Indonésia \\
\hline Petersson et al., 1989 & 1989 & 19 & 53 & CPO-S 29,6 & Suécia \\
\hline Källestal, 1991 & 1987 & 18 & 287 & 6,4 & Suécia \\
\hline Todd \& Lader, 1991 & 1988 & $16-24$ & 706 & $\begin{array}{l}83 \% \text { com } 18 \text { ou }+ \\
\text { dentes hígidos; } 7 \% \\
\text { com } 12 \text { ou }+ \text { dentes } \\
\text { restaurados }\end{array}$ & Reino Unido \\
\hline Rajasuo et al., 1991 & 1991 & $19,8-22,2$ & 353 & 11,2 & Finlândia \\
\hline \multirow[t]{3}{*}{ Antoft et al., 1999} & 1972 & $18-25$ & 1.719 & 16,6 & Dinamarca \\
\hline & 1982 & & 1.442 & 11,8 & \\
\hline & 1993 & & 942 & 6,1 & \\
\hline \multirow[t]{4}{*}{ Hugoson et al., 2000} & 1973 & 20 & 100 & CPO-S 35,1 & Suécia \\
\hline & 1978 & & 100 & CPO-S 27,0 & \\
\hline & 1983 & & 100 & CPO-S 21,5 & \\
\hline & 1993 & & 100 & CPO-S 15,7 & \\
\hline Dawson \& Smales, 1994 & 1994 & $26-30$ & ND & 10,7 & Austrália \\
\hline Asmyhr et al., 1994 & 1994 & 18 & 90 & 10,2 & Noruega \\
\hline Sgan-Cohen et al., 2000 & 1994-1997 & 21 & 7.139 & 8,5 & Israel \\
\hline Kelly et al., 2000 & 1998 & $16-24$ & 491 & 1,6 & Reino Unido \\
\hline
\end{tabular}

ND = Não disponível 


\section{Resultados}

Não houve recusa em participar do estudo. Os valores de kappa para cada um dos dentes variaram entre 0,64 (dente 11) a 1,00 (a maioria dos dentes), tanto para os diagnósticos das condições dentárias, como para a indicação das necessidades de tratamento odontológico, indicando que a concordância intra-examinador foi alta.

A prevalência de cárie foi de $81 \%$ e o índice CPO-D médio foi de 4,5. A média de dentes obturados foi igual a 3,0, o que equivale a dois terços do índice. A observação das distribuições do CPO-D e seus componentes segundo os quartis permite visualizar que a distribuição do índice não é normal (Tabela 2).

$O$ índice CPO-D e seus componentes foram comparados com as variáveis sócio-econômicas de duas formas distintas. Na primeira, estudou-se a ocorrência de cárie, considerandoa ausente $(C P O-D=0)$ ou presente $(C P O-D \geq 1)$. Observa-se que a maior prevalência de ataque de cárie esteve associada com o menor grau de escolaridade das mães e dos pais dos alistandos ( $p<0,001$ ). A prevalência de dentes cariados e perdidos esteve negativamente associada com todas as variáveis sócio-econômicas estudadas ( $p<0,001$ ), enquanto associação entre a prevalência de dentes obturados e indicadores sócio-econômicos não foi associada significativamente (Tabela 3).

A segunda forma de análise foi testar a severidade do ataque de cárie, medida pelo índice CPO-D, em relação às variáveis sócio-econômicas através do Mann-Witney test. $\mathrm{O}$ maior índice CPO-D foi observado nos alistandos Cujos pais e mães apresentavam menor escolari- dade ( $p<0,001$ ). Não houve diferença estatisticamente significativa entre os valores do índice CPO-D e renda familiar. O número de dentes obturados não foi diferente em nenhum dos grupos sócio-econômicos estudados, ao passo que os maiores valores de dentes cariados e perdidos foram encontrados nos indivíduos de menor renda familiar, com menor escolaridade e com pais e mães de menor escolaridade ( $p<0,001$ ) (Tabela 4 ).

Necessidades de tratamento odontológico foram encontradas em $40,7 \%$ da população estudada. Entretanto, apenas 1,2 dente, em média, apresentou-se com necessidade de tratamento, e restaurações simples equivaleram a dois terços das necessidades totais (Tabela 5).

\section{Discussão}

Estudos epidemiológicos de cárie dentária em populações de adultos jovens são raros. Diferentes critérios de diagnóstico e diferentes procedimentos amostrais têm sido utilizados, tornando difícil estabelecer comparações. Por exemplo, no estudo nacional de 1986, foram examinados jovens entre 15 a 19 anos, de ambos os sexos, com os dados apresentados por região e não por município. O nosso estudo abordou apenas jovens de 18 anos, do sexo masculino e de um município da Região Sul. Tendo esses cuidados e observando-se os dados relativos à Região Sul (MS, 1988), podemos considerar que houve uma redução no ataque de cárie no período 1986 a 1999. Em 1986, o CPO-D médio para a faixa etária de 15 a 19 anos da Região Sul foi de 13,8 , sendo no presente estudo igual a 4,5. No estudo de 1986, o

Tabela 2

Estatística descritiva dos componentes cariados, perdidos, obturados, do índice CPO-D e número de dentes presentes em jovens de 18 anos $(n=300)$. Florianópolis, Santa Catarina, Brasil, 1999.

\begin{tabular}{|c|c|c|c|c|c|c|}
\hline & Cariados & Perdidos & Obturados & CPO-D & CPO-D zero & $\begin{array}{c}\text { Dentes } \\
\text { presentes }\end{array}$ \\
\hline Média & 1,2 & 0,3 & 3,0 & 4,5 & - & 28,7 \\
\hline Desvio padrão & 2,0 & 0,9 & 3,2 & 4,01 & - & 1,8 \\
\hline$\%$ & 24,9 & 7,6 & 67,5 & 100,0 & 19,0 & - \\
\hline Mínimo & 0,0 & 0,0 & 0,0 & 0,0 & - & 22,0 \\
\hline Quartil 25\% & 0,0 & 0,0 & 0,0 & 1,0 & - & 28,0 \\
\hline Mediana & 0,0 & 0,0 & 2,0 & 4,0 & - & 28,0 \\
\hline Quartil 75\% & 1,0 & 0,0 & 5,0 & 7,0 & - & 30,0 \\
\hline Máximo & 12,0 & 7,0 & 16,0 & 20,0 & - & 32,0 \\
\hline
\end{tabular}


Associação entre ocorrência de dentes cariados $(C \geq 1)$, dentes perdidos por cárie $(P \geq 1)$, dentes obturados $(O \geq 1)$ e prevalência de cárie (CPO-D $\geq 1)$ e variáveis sócio-econômicas em jovens de 18 anos ( $n=300)$. Florianópolis, Santa Catarina, Brasil, 1999.

\begin{tabular}{|c|c|c|c|c|c|c|c|c|}
\hline \multirow[t]{2}{*}{ Variáveis } & \multicolumn{2}{|c|}{ Cariados } & \multicolumn{2}{|c|}{ Perdidos } & \multicolumn{2}{|c|}{ Obturados } & \multicolumn{2}{|c|}{ CPO-D } \\
\hline & \multicolumn{2}{|c|}{ n (\%) } & \multicolumn{2}{|c|}{$\mathrm{n}(\%)$} & \multicolumn{2}{|c|}{ n (\%) } & \multicolumn{2}{|c|}{ n (\%) } \\
\hline Renda familiar & \multicolumn{2}{|c|}{$p<0,001$} & \multicolumn{2}{|c|}{$p<0,001$} & \multicolumn{2}{|c|}{$P=0,16(N S)$} & \multicolumn{2}{|c|}{$P=0,13(N S)$} \\
\hline$<5 \mathrm{SM}$ & $30(38,5)$ & $48(61,5)$ & $51(65,4)$ & $27(34,6)$ & $28(35,9)$ & $50(64,1)$ & $8(10,3)$ & $70(89,7)$ \\
\hline$\geq 5 \mathrm{SM}$ & $135(64,6)$ & $74(35,4)$ & $180(86,1)$ & $29(13,9)$ & $57(27,3)$ & $152(72,7)$ & $38(18,2)$ & $171(81,8)$ \\
\hline $\begin{array}{l}\text { Escolaridade } \\
\text { do jovem }\end{array}$ & \multicolumn{2}{|c|}{$p<0,001$} & \multicolumn{2}{|c|}{$p<0,001$} & \multicolumn{2}{|c|}{$p=0,26(N S)$} & \multicolumn{2}{|c|}{$p=0,07(N S)$} \\
\hline$\leq 8$ anos & $52(40,9)$ & $75(59,1)$ & $87(68,5)$ & $40(31,5)$ & $42(33,1)$ & $85(66,9)$ & $15(11,8)$ & $112(88,2)$ \\
\hline$>8$ anos & $116(71,2)$ & $47(28,8)$ & $146(89,6)$ & $17(10,4)$ & $44(27,0)$ & $119(73,0)$ & $32(19,6)$ & $131(80,4)$ \\
\hline $\begin{array}{l}\text { Escolaridade do pai } \\
\text { do jovem }\end{array}$ & \multicolumn{2}{|c|}{$p<0,001$} & \multicolumn{2}{|c|}{$p<0,001$} & \multicolumn{2}{|c|}{$p=0,84(N S)$} & \multicolumn{2}{|c|}{$p<0,001$} \\
\hline$\leq 8$ anos & $61(43,6)$ & $79(56,4)$ & $101(72,1)$ & $39(27,9)$ & $42(30,0)$ & $98(70,0)$ & $15(10,7)$ & $125(89,3)$ \\
\hline$>8$ anos & $101(74,8)$ & $34(25,2)$ & $123(91,1)$ & $12(8,9)$ & $39(28,9)$ & $96(71,1)$ & $31(23,0)$ & $104(77,0)$ \\
\hline $\begin{array}{l}\text { Escolaridade da mãe } \\
\text { do jovem }\end{array}$ & \multicolumn{2}{|c|}{$p<0,001$} & \multicolumn{2}{|c|}{$p<0,001$} & \multicolumn{2}{|c|}{$p=0,90(N S)$} & \multicolumn{2}{|c|}{$p<0,001$} \\
\hline$\leq 8$ anos & $81(48,8)$ & $85(51,2)$ & $120(72,3)$ & $46(27,7)$ & $50(30,1)$ & $116(69,9)$ & $19(11,4)$ & $147(88,6)$ \\
\hline$>8$ anos & $85(71,4)$ & $34(28,6)$ & $111(93,3)$ & $8(6,7)$ & $35(29,4)$ & $84(70,6)$ & $28(23,5)$ & $91(76,5)$ \\
\hline
\end{tabular}

NS = não significante; $\mathrm{SM}$ = salário mínimo.

Nota: Excluídos os ignorados.

componente obturado do índice foi igual a 5,9, o que equivale a $46,4 \%$ do índice no Brasi I, e 7,1 $(51,4 \%)$ na Região Sul, revelando que aproximadamente metade dos dentes atacados pela cárie estava sem tratamento. Os maiores índices de dentes cariados concentraram-se nos grupos de menor renda, revelando que as condições de saúde bucal expressam as desigualdades sociais.

Ao se comparar o índice CPO-D da população do nosso estudo $(4,5)$ com o obtido para a população de 12 anos do município $(2,7)$ (Peres et al., 1996), verifica-se um incremento anual de 0,36 dente atacado pela cárie, o que pode ser considerado baixo. Hipoteticamente, o menor número de dentes atacados pela cárie pode ter possibilitado uma maior cobertura da população pelos serviços odontológicos neste período. O comportamento do componente obturado do índice CPO-D aumentou no período compreendido entre 1986 e 1999.

A prevalência de cárie dentária encontrada (81\%) assemelha-se às encontradas na Austrália (Dale, 1969), na Finlândia em 1991 (Rajasuo et al., 1991), na Inglaterra em 1995 (Richardson \& Mclntyre, 1996), todas elevadas. No entanto, foi maior que a prevalência de $51 \%$ registrada no Reino Unido, entre 16 a 24 anos de idade, em 1998 (Kelly et al., 2000).
O número mínimo de dentes presentes na população estudada foi de 22 dentes, ou seja, todos os al istandos da amostra apresentaram mais de vinte dentes presentes. Um número mínimo de vinte dentes naturais, incluindo os incisivos, caninos e pré-molares, satisfazem a maioria dos critérios para um aceitável nível de saúde bucal (Käyser, 1984). A porcentagem da população estudada com 28 dentes ou mais foi igual a $82,5 \%$. A presença de $85 \%$ da população de 18 anos com todos os dentes naturais presentes é a meta estabelecida pela OMS/ Federação Dentária Internacional para o ano 2000 (FDI, 1982).

O quadro epidemiológico favorável observado neste estudo pode ser devido às características particulares do município. Deve-se considerar que Florianópolis apresenta elevados indicadores sociais quando comparados com os de outros municípios brasileiros. O município apresenta-se com os melhores indicadores sociais (dados de 1996) dentre todas as capitais brasileiras, sendo o segundo município brasileiro em qualidade de vida (PNUD, 1998).

As menores médias do índice CPO-D, assim como as menores prevalências de cárie concentraram-se nos grupos de menor escolaridade. A escolaridade mostrou-se um melhor indicador sócio-econômico do que a renda fami- 
Tabela 4

Índice CPO-D e componentes, segundo variáveis sócio-econômicas em jovens de 18 anos ( $n=300)$.

Florianópolis, Santa Catarina, Brasil, 1999.

\begin{tabular}{|c|c|c|c|c|}
\hline Variáveis & $\begin{array}{c}\text { Cariados } \\
\text { Média (erro padrão) }\end{array}$ & $\begin{array}{c}\text { Perdidos } \\
\text { Média (erro padrão) }\end{array}$ & $\begin{array}{c}\text { Obturados } \\
\text { Média (erro padrão) }\end{array}$ & $\begin{array}{c}\text { CPO-D } \\
\text { Média (erro padrão) }\end{array}$ \\
\hline Renda familiar & $p<0,001$ & $p<0,001$ & $p=0,19(N S)$ & $p=0,24$ (NS) \\
\hline$<5 \mathrm{SM}$ & $1,9(0,3)$ & $0,5(0,1)$ & $2,7(0,3)$ & $5,1(0,5)$ \\
\hline$\geq 5 \mathrm{SM}$ & $0,9(0,1)$ & $0,3(0,0)$ & $3,3(0,2)$ & $4,3(0,3)$ \\
\hline Total & $1,2(0,1)$ & $0,3(0,0)$ & $3,0(0,2)$ & $4,5(0,2)$ \\
\hline Escolaridade do jovem & $p<0,001$ & $p<0,001$ & $p=0,43(N S)$ & $p<0,001$ \\
\hline$\leq 8$ anos & $2,0(0,2)$ & $0,6(0,1)$ & $3,0(0,3)$ & $5,6(0,4)$ \\
\hline$>8$ anos & $0,5(0,0)$ & $0,2(0,0)$ & $3,1(0,2)$ & $3,9(0,3)$ \\
\hline Total & $1,6(0,1)$ & $0,3(0,0)$ & $3,1(0,2)$ & $4,7(0,2)$ \\
\hline $\begin{array}{l}\text { Escolaridade do pai } \\
\text { do jovem }\end{array}$ & $p<0,001$ & $p<0,001$ & $p=0,94(N S)$ & $p<0,001$ \\
\hline$\leq$ Até 8 anos & $1,8(0,2)$ & $0,5(0,0)$ & $3,1(0,3)$ & $5,4(0,3)$ \\
\hline$>8$ anos & $0,5(0,0)$ & $0,2(0,0)$ & $3,2(0,3)$ & $3,9(0,3)$ \\
\hline Total & $1,1(0,1)$ & $0,3(0,0)$ & $3,2(0,2)$ & $4,6(0,2)$ \\
\hline $\begin{array}{l}\text { Escolaridade da mãe } \\
\text { do jovem }\end{array}$ & $p<0,001$ & $p<0,001$ & $p=0,88(N S)$ & $p<0,001$ \\
\hline$\leq 8$ anos & $1,6(0,2)$ & $0,5(0,0)$ & $3,1(0,2)$ & $5,2(0,4)$ \\
\hline$>8$ anos & $0,6(0,1)$ & $0,0(0,0)$ & $3,2(0,3)$ & $3,8(0,3)$ \\
\hline Total & $1,1(0,1)$ & $0,3(0,0)$ & $3,1(0,2)$ & $4,5(0,2)$ \\
\hline
\end{tabular}

Valores de $\mathrm{p}=$ Mann-Whitney test.

$\mathrm{SM}=$ salário mínimo; NS = não significante.

Tabela 5

Necessidades de tratamento odontológico em jovens de 18 anos $(n=300)$. Médias de dentes por pessoa, erro padrão (EP) e porcentagens. Florianópolis, Santa Catarina, 1999.

\begin{tabular}{lccccc}
\hline Parâmetros & $\begin{array}{c}\text { Restauração } \\
\text { (1 superfície) }\end{array}$ & $\begin{array}{c}\text { Restauração (2 } \\
\text { superfícies ou +) }\end{array}$ & $\begin{array}{c}\text { Prótese (2 ou } \\
\text { + elementos) }\end{array}$ & $\begin{array}{c}\text { Exodontia } \\
\text { Total } \\
\text { restauração }\end{array}$ \\
\hline Média & 0,4 & 0,4 & 0,0 & 0,2 & 0,2 \\
EP & 0,8 & 1,0 & 0,1 & 0,8 & 0,6 \\
$\%$ & 33,3 & 33,3 & 0,0 & 1,2 & 16,7 \\
\hline
\end{tabular}

liar neste estudo. Essa também é a opinião de Borrel (1997), quando argumenta que a educação dá acesso a uma determinada ocupação, e, portanto, a um certo nível de renda, e isso pode influenciar o acesso a diferentes condutas relacionadas à saúde. Por essa razão, escolaridade é um dos indicadores mais utilizados para aferir condição sócio-econômica em epidemiologia.

As desi gualdades em saúde bucal, por conseguinte, persistem, apesar dos índices mais favoráveis encontrados neste estudo, quando comparados com o de 1986, e dos bons indicadores sociais do município. Vale lembrar que, no estudo de 1986, também foi estabelecido o percentual de jovens que apresentavam todos os dentes, segundo estrato de renda. Os resultados mostraram que $26 \%$ dos jovens com renda até dois salários mínimos e $36 \%$ daqueles com três a quatro salários mínimos apresentaram todos os dentes presentes, enquanto $57 \%$ do extrato de renda de cinco ou mais salários 
mínimos apresentaram todos os dentes (MS, 1988).

Alguns aspectos metodológicos deste estudo merecem registro. Estudos epidemiológicos com jovens, que permitam inferências populacionais, são operacionalmente difíceis, pois idealmente devem ser de base domiciliar. Neste estudo, utilizou-se a lista de jovens que se apresentaram para o alistamento militar do Exército Brasileiro em Florianópolis. Para comparar as características da população do nosso estudo com as da população em geral, foi analisada a distribuição do grau de escolaridade da população de Florianópolis (IBGE, 1996) com a dos pais dos alistandos. Esta comparação mostrou grande semelhança. Os dados do IBGE (Fundação Instituto Brasileiro de Geografia e Estatística) mostraram que, em 1996, 36\% dos homens e $46,4 \%$ das mulheres entre 20 e 64 anos de idade tinham até oito anos de estudo, comparados com $37,4 \%$ dos pais dos alistandos e $44,9 \%$ das mães dos alistandos do presente estudo. Em razão dessas semelhanças, a relação de alistandos do Exército pode ser uma boa al ternativa para estudos epidemiológicos em saúde bucal que objetivem inferir os resultados para a população masculina de 18 anos de idade. Entretanto, mantém-se a limitação de se desconhecer como é o padrão epidemiológico da população feminina da mesma idade, cujos acesso a serviços e comportamentos são hipoteticamente distintos dos observados no sexo masculino.

As necessidades de tratamento odontológico da população estudada podem ser consideradas de simples resolução, mediante procedimentos clínico-cirúrgicos condizentes com o nível primário de atenção.

Pode-se concluir que o quadro epidemiológico da população de jovens de 18 anos do sexo masculino em Florianópolis é favorável. Porém, os grupos de menor renda e menor escolaridade concentram a maior parte da doença não tratada, devendo receber ações preventivas e assistenciais prioritárias.

\section{Referências}

ANTOFT, P.; RAMBUSCH E.; ANTOFT, B. \& CHRISTENSEN, H. W., 1999. Caries experience, dental health behaviour and social status - Three comparative surveys among Danish military recruits in 1972, 1982 and 1993. Community Dental Health, 16:80-84.

ASMYHR, O.; GRYTTEN, L. \& GRYTTEN, J., 1994. Changing trends in caries experience among male military recruits in Norway. Community Dentistry Oral Epidemiology, 22:206-207.

BORREL, C., 1997. Métodos utilizados no estudo das desigualdades sociais em saúde. In: Condições de Vida eSituação de Saúde (R. B. Barata, org.), pp. 167-196, Rio de Janeiro: ABRASCO.

DALE, J. W., 1969. Prevalence of dental caries and periodontal disease in military personnel. Australian Dental Journal, 14:30-37.

DePAOLA, P. F., 1983. The Massachusetts health survey. Journal of the Massachussetts Dentistry Society, 32:10-11, 23-25.

DAWSON, A. S. \& SM ALES, R. J., 1994. Dental health changes in an Australian Defense Force Population. Australian Dental Journal, 39:242-246. 
FDI (Federation Dentaire Internationale), 1982. Global goals for oral health in the year 2000. International Dental Journal, 32:74-77.

GAARE, D.; JOELIMAR, F. A.; OUDERAA, F. V. \& ROLLA G. A., 1989. Cross sectional study of DMFT and CPITN scores in a group of Indonesian soldiers. Scandinavian Journal of Dental Research, 97:2024.

GESSER, H. C.; PERES, M. A. \& MARCENES, W., 2001. Prevalência de condições gengivais e periodontais associadas a fatores sócio-econômicos. Revista de Saúde Pública, 35:289-293.

HUGOSON, A.; KOCH, G.; HALOONSTEN, A. L.; NORDERYD, J. \& ABERG, A. 2000. Caries prevalence and distribution in 3-20-years-olds in Jönköping, Sweden, in 1973, 1978, 1983, and 1993. Community Dentistry and Oral Epidemiology, 28:83-89.

IBGE (Fundação Instituto Brasileiro de Geografia e Estatística), 1996. Contagem da População de 1996. v. 2. Resultados Relativos à Grande Florianópolis (SC). Rio de Janeiro: IBGE.

KÄLLESTAL, C., 1991. Dental caries in 16 and 18 year old adolescents in northern Sweden. Scandinavian Journal of Dental Research, 99:100-105.

KÄYSER, A. F., 1984. Minimum number of teeth needed to satisfy functional and social demands. In: Public Aspects of Periodontal Disease (A. Frandsen, ed.), p. 135, Chicago: Quintessence Books.

KELLY, M.; STEELE, J.; NUTTALL, N.; BRADNOCK, G.; MORRIS, J.; NUNN, J.; PINE, C.; PITTS, N.; TRASURE, E. \& WHITE, D., 2000. Adult Dental Health Survey. Oral Health in the United Kingdom 1998. London: The Stationery Office.

MORGAN, M. V.; STONNILL, A. \& LASLETT, A. M., 1992. Dental caries amongst Royal Australian Navy recruits. Australian Dental Journal, 37:201-204.

MS (Ministério da Saúde), 1988. Levantamento Epidemiológico em Saúde Bucal: Brasil I, Zona Urbana, 1986. Brasília: Centro de Documentação, MS.

PERES, M. A. A.; FREITAS, S. T.; LACERDA, J. \& CALVO, M. C. M., 1996. Prevalência de cárie dentária na população de 3 a 12 anos de idade do Município de Florianópolis, SC, Brasil, 1995. In: XIV Encontro Nacional de Técnicos e Administradores de Serviços Públicos Odontológicos. I Congresso Brasileiro de Saúde Bucal Coletiva, Cadernos de Resumos, p. 31. Curitiba: Secretaria Municipal de Saúde.
PERES, M. A. A.; TRAEBERT, J. L. \& MARCENES, W., 2001. Calibração de examinadores para estudos epidemiológicos de cárie dentária. Cadernos de Saúde Pública, 17:153-159.

PETERSSON, L. G.; JONSSON, G.; STADLER, L. E.; SAMFORS, K. A. \& GLEERUP, A., 1989. Oral status and estimated treatment need in Swedish air force conscripts. Swedish Dental Journal, 13:69-76.

PNUD (Programa da Nações Unidas para o Desenvolvimento) 1998. Desenvolvimento Humano. Condições deVida. Indicadores Sociais. Brasília: PNUD.

RAJASUO, A.; MURTOMAA, H.; MEURMAN, J. H. \& ANKKURINIEMI, O., 1991. Oral health problems in Finnish conscripts. Military Medicine, 156:16-18.

RICHARDSON, P. S. \& McINTYRE, I. G., 1996. Dental treatment needs of a cohort of Royal Air Force recruits over 5 years. Community Dental Health, 13:11-16.

SGAN-COHEN, H. D.; KATZ, J.; HOREV, T.; DINTE, H. \& ELDAD, A., 2000. Trends in caries and associated variables among Israeli adults over 5 decades. Community Dentistry and Oral Epidemiology, 28:234-240.

TODD, J. E. \& LADER, D., 1991. Adult Dental Health in the United Kingdom in 1988. London: Office of Population Censuses and Surveys.

TRUIN, G. . .; KONING, K. G. \& KALSBEEK, H., 1993. Trends in dental caries in The Netherlands. Advanced Dental Research, 7:15-18.

VON DER FEHR, F. R., 1982. Evidence of decreasing caries prevalence in Norway. Journal of Dental Research, 61(Special Issue):1356-1360.

WHO (World Health Organization), 1997. Oral Health Surveys. Basic Methods. 4th Ed. Geneva: WHO.

Recebido em 22 de fevereiro de 2001

Versão final reapresentada em 13 de setembro de 2001 Aprovado em 28 de novembro de 2001 